\title{
1 Oxytocin receptor expression patterns in the human brain across
}

\section{2 development}

4 Jaroslav Rokicki ${ }^{1,2.3}$, Tobias Kaufmann ${ }^{1,4}$, Ann-Marie G. De Lange ${ }^{1,5,6}$, Dennis van der Meer $5 \quad{ }^{1,7}$, Shahram Bahrami ${ }^{1,2}$, Alina M. Sartorius ${ }^{1,2}$, Unn K. Haukvik ${ }^{1,3}$, Nils Eiel Steen ${ }^{1}$, Emanuel 6 Schwarz $^{8}$, Dan J. Stein ${ }^{9}$, Terje Nærland ${ }^{10,11}$, Ole A. Andreassen ${ }^{1,11}$, Lars T. Westlye ${ }^{1,2,11}$, 7 and Daniel S. Quintana ${ }^{1,2,11^{*}}$

$9 \quad{ }^{1}$ NORMENT Centre for Psychosis Research, Division of Mental Health and Addiction, University of 10 Oslo and Oslo University Hospital, Oslo, Norway

$11{ }^{2}$ Department of Psychology, University of Oslo, Oslo, Norway

$12{ }^{3}$ Centre of Research and Education in Forensic Psychiatry, Oslo University Hospital, Oslo, Norway

$13{ }^{4}$ Department of Psychiatry and Psychotherapy, University of Tübingen, Germany

$14{ }^{5}$ LREN, Centre for Research in Neurosciences - Department of Clinical Neurosciences, CHUV and 15 University of Lausanne, Lausanne, Switzerland

$16{ }^{6}$ Department of Psychiatry, University of Oxford, Oxford, UK

$17{ }^{7}$ School of Mental Health and Neuroscience, Faculty of Health, Medicine and Life Sciences, Maastricht 18 University, Maastricht, The Netherlands

$19{ }^{8}$ Central Institute of Mental Health, Department of Psychiatry and Psychotherapy, Medical Faculty 20 Mannheim, Heidelberg University, Mannheim, Germany

219 SAMRC Unit on Risk \& Resilience in Mental Disorders, Department of Psychiatry and Neuroscience 22 Institute, University of Cape Town, Cape Town, South Africa

$23 \quad{ }^{10}$ NevSom, Department of Rare Disorders, Oslo University Hospital, Oslo, Norway

$24{ }^{11}$ KG Jebsen Centre for Neurodevelopmental Disorders, University of Oslo, Oslo, Norway

27 * Corresponding author: Daniel S. Quintana (daniel.quintana@psykologi.uio.no) 
Abstract

Background: Oxytocin plays a vital role in social behavior and homeostatic processes, with animal models indicating that oxytocin receptor (OXTR) expression patterns in the brain influence behavior and physiology. However, the developmental trajectory of OXTR gene expression is unclear, which is considerable knowledge gap as many psychiatric illnesses emerge early in life and genes tend to be differentially regulated across development.

Methods: We calculated spatiotemporal expression patterns for 16660 genes in the human brain using transcriptome data from 57 donors across the lifespan. Next, we explored the evolutionary origin of these patterns using a comparative gene expression dataset from 38 macaque donors. Finally, we determined the functional significance of OXTR spatiotemporal co-expression patterns via the annotation of genetic associations with psychiatric and body composition phenotypes.

Results: OXTR expression in the brain accelerated before birth with a peak in early childhood and OXTR expression was highly correlated with dopamine receptor D2 expression across development. These gene expression patterns were not observed in a comparative macaque sample, suggesting they might be evolutionarily new features. In addition, a network of genes strongly spatiotemporally coupled with OXTR was enriched in schizophrenia, cognitive, and body composition phenotypes, with elements of this gene network having undergone positive selection in humans but not macaques.

Conclusions: These results demonstrate that oxytocin signaling plays an important role in a diverse set of psychological and physiological processes across the lifespan. Identifying a critical window of OXTR expression in the brain, together with associated genes, may help illuminate our understanding of disease etiology. 
Oxytocin is an evolutionarily ancient neuromodulator that facilitates mammalian social behavior and metabolic regulatory processes $(1,2)$. Oxytocin is primarily produced in the hypothalamus, for both central and peripheral release (3), and binds to oxytocin receptors that are located throughout the brain and the periphery $(1,2)$. Both the experimental manipulation of OXTR expression levels and OXTR gene knockout can have a dramatic effect on behavior and physiology $(4,5)$. Thus, the identification of OXTR expression patterns in the human brain can help identify the functional relevance of the oxytocin signaling system. We recently mapped the voxel-wise density of OXTR expression across the adult human brain, finding increased expression in subcortical and olfactory regions (6). Moreover, the location of OXTR expression was highly correlated with dopaminergic gene expression and closely matched the neural activity patterns observed in anticipatory, appetitive, and aversive mental states, along with homeostatic regulation. Research has also shown that compared to control donors, OXTR expression in the dorsolateral prefrontal cortex is increased in donors diagnosed with mood disorders (7) and reduced in the temporal cortex of donors diagnosed with schizophrenia (8).

While the OXTR gene expression pattern in the adult human brain has been described (6), little is known about the evolution and functional relevance of OXTR gene expression and OXTR gene co-expression patterns across the lifespan. This is a critical knowledge gap, as genes are differentially regulated across the brain over development (9) and disturbances in neural development contribute to the genesis of mental illness (10). Supporting the clinical relevance of spatially and temporally distinct aberrations, neurotypical postmortem brain tissue from the ventral pallidum (VP) and nucleus basalis of Maynert has been reported to have higher OXTR binding than postmortem VP tissue from autistic donors, and an early childhood peak of OXTR binding that was observed in VP tissue from neurotypical donors was absent in VP tissue from autistic donors (11).

The oxytocin system emerges very early in the course of mammalian development. Oxytocin has been detected prenatally during the neurogenesis period of fetal brain 
development (12) and magnocellular oxytocin neurons only mature a few weeks postnatally (13). Oxytocin system dysregulation during early development also plays an important role in behavior later in life (14). For example, oxytocin concentrations in cerebrospinal fluid (CSF) are significantly higher in mother-reared rhesus monkeys compared to nursery reared monkeys (15) and increased maternal behaviors are associated with higher central OXTR expression levels in rat pups (16). In humans, women with a history of child abuse also demonstrate reduced CSF oxytocin (17), highlighting how the early environment can influence later oxytocin system functioning.

Mental and physical illnesses tend to follow specific developmental trajectories. Thus, the identification of a critical window of oxytocin system expression in the brain, together with associated genes, may illuminate our understanding of mental illnesses. Research has identified patterns of increased OXTR expression in non-human mammals during either infancy, childhood, or adulthood, compared to other lifespan periods (18). However, it is not known which of these characteristic patterns are observed in humans and the functional significance of observed patterns. In addition, some aspects of oxytocin's role in behavior are sexually dimorphic, but it is unclear whether sex differences in gene expression patterns are related to these effects. Therefore, there is a need to characterize typical oxytocin pathway system development, to determine whether expression patterns are stable over development, and to identify critical periods.

Here we identified the spatiotemporal distribution patterns of OXTR expression and gene expression interactions in human brain tissue from the prenatal period to late adulthood. We also examined the evolutionary conservation of OXTR expression patterns by replicating our first analysis using macaque OXTR expression data and extended this analysis by identifying the evolutionary origin of genes with strong spatiotemporal associations with OXTR expression. Finally, we explored the functional significance of genes with a strong spatiotemporal association with OXTR by assessing if this geneset was enriched of in various GWAS of psychiatric, cognitive, and body composition phenotypes. 
To better understand the functional relevance of spatiotemporal gene co-expression patterns across the lifespan and relevance to common human traits and diseases, we determined OXTR expression patterns, along with all available protein coding genes, across sixteen regions of the human brain from the prenatal stage (5.7 pre-conception weeks) to 82 years of age in 57 donors (26 females, 31 males). We used genome-wide exon-level transcriptome data available from the

Gene

Expression

Omnibus

database (https://www.ncbi.nlm.nih.gov/geo; series GSE25219) as a proxy of OXTR receptor density (19). Human gene expression data was converted from months and years into days for the purposes of analysis.

Gene expression values for sixteen brain regions were visualized across the lifespan using two approaches. The first approach illustrates absolute change in gene expression patterns across the lifespan for sixteen brain regions. We analyzed data from sixteen brain regions in humans: primary motor cortex (M1C), dorsolateral prefrontal cortex (DFC), ventrolateral prefrontal cortex (VFC), orbital frontal cortex (OFC), primary somatosensory cortex (S1C), inferior parietal cortex (IPC), primary auditory cortex (A1C), caudal superior temporal cortex (STC), inferolateral temporal cortex (ITC), primary visual cortex (V1C), medial prefrontal cortex (MFC), hippocampus (HIP), striatum (STR), amygdala (AMY), mediodorsal nucleus of the thalamus (MD), and cerebellar cortex (CBC). The expression values were smoothed via a logarithmic scale using locally weighted least squares regression, then demeaned and scaled (i.e., divided by the standard deviation), yielding a data frame with expression values converted to Z-scores per brain region of interest. Thus, the mean expression of a given brain region across the lifespan is zero. In these "ribbon plots", Z-values are stacked on top of each other and scaled again by number of regions, so when a single region (or group of regions) demonstrates increased expression relative to other life periods, the peaks will be higher. 

plot", with the key difference of age normalized expression values. This provides a stronger emphasis on expression changes in specific brain regions, rather than overall expression across the brain. For example, a value of 0.25 in the cerebellum during adolescence means that at this developmental stage $25 \%$ of gene expression occurs in the cerebellum compared to $75 \%$ in the remaining 15 regions at the same age period. Expression levels are shown by colors instead of ribbon height.

\section{Lifespan correlation between genes}

We have previously shown using a voxel-wise approach in the adult brain that OXTR expression is highly correlated with the expression of a selection of oxytocinergic, dopaminergic, muscarinic acetylcholine, and opioid pathway genes (6). Thus, we assessed whether this geneset also had a strong spatiotemporal relationship with OXTR. To calculate the lifespan correlation for two given genes, we first we calculated Spearman's $r$ correlation coefficient within each donor. Then, we interpolated calculated points on a logarithmic scale using locally weighted least squares regression (with a span of 0.4 for the human data and a span of 0.7 for the macaque data). To evaluate the lifespan average, we calculated the mean of the interpolated line, which we labeled the "trajectory" mean. By using this approach, each life period receives an equal weighting. To complement this measure, we also presented the

Using correlation data between genes, we generated a $14 \times 14$ correlation matrix reflecting the spatiotemporal Spearman's correlation for each donor for each mRNA map pair, using the mean of fitted trajectories in lower-left triangle, so that each time period gets equal 
weight and mean of all points in the upper-right triangle, so that each subject gets equal weight. As per our previous analysis (6), we used the following genes (Oxytocin pathway set: OXTR, CD38, OXT; Dopaminergic set: DRD1, DRD2, DRD3, DRD4, DRD5, COMT, and DAT1; muscarinic acetylcholine set: CRHM1, CRHM2, CRHM3, CRHM4, and CRHM5; opioid set: OPRM1, OPRD1, OPRK1, and AVPR1A, which is a vasopressin signaling gene). We used Ward's hierarchical clustering to identify highly correlated groups of genes within this geneset.

\section{The evolutionary origin of genes spatiotemporally associated with OXTR}

To explore the degree to which spatiotemporal patterns of oxytocin pathway expression across human development reflect a recent evolutionary adaption, rhesus macaque (macaca mulatta) gene expression data from post-mortem brain tissue was extracted from the National Institute of Health Blueprint Non-human primate (NIH Blueprint NHP) atlas (http://www.blueprintnhpatlas.org/). This atlas provides data for central gene expression across six prenatal stages $(40,50,70,80,90$, and 120 embryonic days) and four postnatal periods (neonate, infant, juvenile, and young adult). We assumed pregnancy to last 40 weeks for humans and 166.5 embryonic days for macaques. Spatiotemporal OXTR expression patterns in macaques were generated using the same methods as described above as humans (i.e., a ribbon plot to best illustrate change across time and heat plot to best illustrate changes in specific brain regions). For the macaque analysis, twenty brain regions were analyzed.

While comparing gene expression patterns between humans and macaques is instructive, this only represents a comparison within a relatively short evolutionary period for two species. In contrast, phylostratigraphy can facilitate the identification of the evolutionary origin of genes using data across the entire evolutionary tree (20). As gene modules with high co-expression in the brain are related to molecular functions (21), we created a geneset containing the genes with the 100 strongest spatiotemporal correlations with OXTR. For our 
phylostratigraphy analysis, a phylostratigraphic map from Domazet-Lošo and Tautz (22), with gene age inferences combined with human gene expression data from five ontogenetic stages (prenatal, infant, child, adolescent, adult) extracted from the BrainSpan atlas (http://brainspan.org) for evolutionary transcriptomics via the 'myTAl' R package (23). For each brain region, a transcriptional age index (TAl) was calculated for each ontogenetic stage by calculating the average age of genes that contribute to the transcriptome (20). To compute the TAI, the expression level for each gene is multiplied by its gene age (i.e., its phylogenetic stage), and then the values for each gene in a geneset are averaged. As older phylostrata are associated with the genesis of a higher number of genes, the TAl provides more weight to genes from younger phylostrata. Altogether, lower values represent an older transcriptome age. In addition, the evolution of protein coding genes in humans and macaques was assessed by comparing their genetic sequences to their common primate ancestor via the GenEvo tool (24). From this data, the numbers of non-synonymous changes per nonsynonymous sites (dN) and synonymous changes per synonymous sites (dS) were computed to calculate the $\mathrm{dN} / \mathrm{dS}$ ratio to estimate the conservation of genes. Welch's $t$-test was used to compare the $\mathrm{dN} / \mathrm{dS}$ ratio of the macaque and human genesets.

\section{The functional significance of genes spatiotemporally associated with OXTR}

To explore the functional significance of spatiotemporal expression patterns of genes strongly coupled with OXTR, a geneset including the top 100 genes with the strongest spatiotemporal correlations with OXTR were submitted to FUMA for annotation of genetic associations (25). Hypergeometric tests (Benjamini-Hochberg adjusted) were performed to examine if this geneset was overrepresented in GWAS from the National Human Genome Research InstituteEuropean Bioinformatics Institute (NHGRI-EBI) GWAS catalogue (e96 2019-09-24; (26)) and biological processes from the Molecular Signatures database (MsigDB v7.0; (27)). We also calculated the top 20 correlated and anti-correlated genes between OXTR and the 16659 
remaining genes across the lifespan, ordered by the lifespan trajectory mean using methods described above.

To assess if there was a significant difference in the association between the spatiotemporal expression of OXTR and genes that have been associated with psychiatric and physiological phenotypes of interest, we compared the distribution of correlation coefficients between phenotype genesets of interest against all remaining genes. We selected a range of psychiatric and psychological phenotypes that have been previously associated with oxytocin signaling dysfunction [i.e., schizophrenia (SCZ), major depressive disorder (MDD), IQ, general cognition, bipolar disorder (BD), autism spectrum disorder (ASD), and anorexia nervosa], and a set of physiological phenotypes based on results from analysis above (i.e., bone fracture, bone density, and BMI). To retrieve genesets associated with phenotypes of interest we performed genome-wide gene-based association using MAGMA (v1.08) and functional mapping of variants to genes based on expression quantitative trait loci (eQTL) via FUMA on the complete GWAS input data available from public resources (See Supplementary table 1 for details). All variants in the GWAS outside of the MHC region (chr6:28,477,797-33,448,354) were included to estimate the significance value of that gene. The eQTL approach maps SNPs to genes which are likely to affect the expression of those genes up to 1 megabase away from the SNP of interest. To determine gene expression and assess eQTL functionality of likely regulatory SNPs, we used data from the eQTL Catalogue (28), PsychENCODE (29), the xQTLServer (30), the CommonMind Consortium (31), GTEx v8 (32), and the Braineac eQTLs dataset (33). MAGMA performs multiple linear regression to obtain gene-based $p$-values and the Bonferroni-corrected significant thresholds for each phenotype is listed in Supplementary table 2. After retrieving genes, we computed Spearman's correlation coefficients to estimate the spatiotemporal relationship between OXTR and 16661 available genes. We then created a distribution for these correlation coefficients. Finally, genes were split into two parts, ones belonging to phenotype of interest and the remaining genes, which were superimposed for comparison. We used non-parametric Mann-Whitney U 
tests to test if distributions had different medians. We adjusted reported $p$-values to the total number of brain phenotypes using a false discovery rate (FDR) threshold.

\section{Donor-to-donor reproducibility of gene expression patterns}

244 To calculate spatiotemporal differential stability, each of the 57 human donors were matched to their three closest three neighbors by age, with duplicates and matches with less than 4 regions in common removed, yielding a list with 93 matches. Each donor was matched 3.2 times, on average. We iterated through all the 131 matched pairs, performing the following tasks for each protein coding gene in the database to calculate spatiotemporal differential stability: 1) Extraction of gene expression for all available brain regions, 2) Calculation of Spearman's correlation coefficient, 3) Calculation of the mean Spearman's correlation coefficient among the 109 pairs.

\section{Data and code availability}

The $\mathrm{R}$ code to recreate our analyses and figures, along with links to the public data used in our analyses, are available at https://gitlab.com/jarek.rokicki/spatio-temporal-oxytocin/.

\section{Results}

\section{Spatiotemporal oxytocin receptor expression in humans and macaques}

Our analysis revealed that OXTR expression across the brain in humans begins to accelerate just before birth, with a peak level of expression occurring during early childhood (Fig. 1a), consistent with previously reported OXTR receptor binding temporal patterns from the ventral pallidum (11). Regional analyses demonstrated increased OXTR expression in the mediodorsal nucleus of the thalamus during early childhood (Fig. 1b) and in the cerebellar cortex and medial prefrontal cortex in later childhood. During adulthood, expression increased 


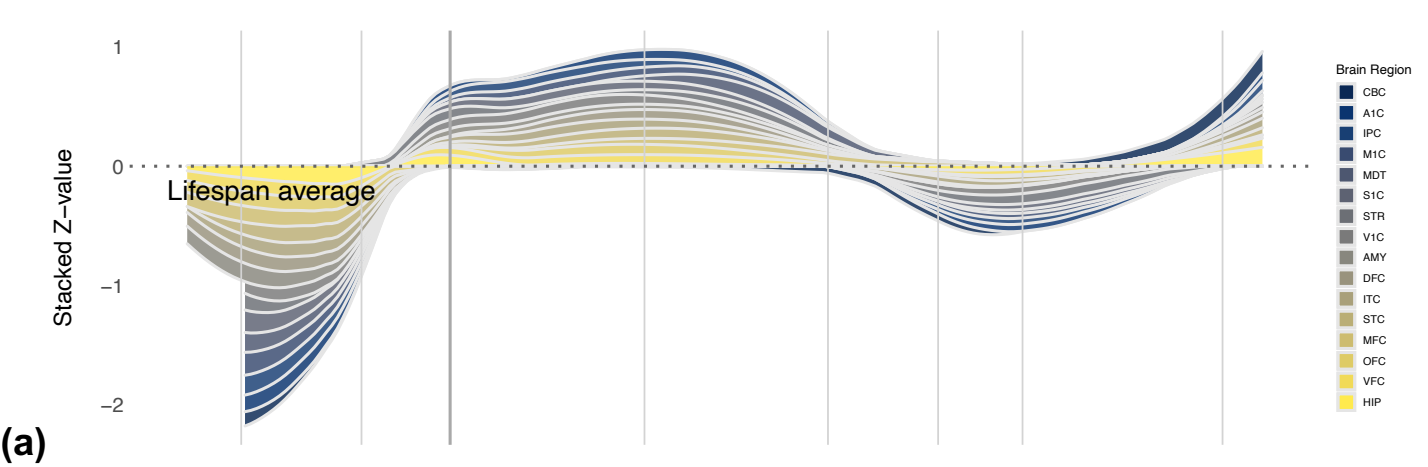

(a)

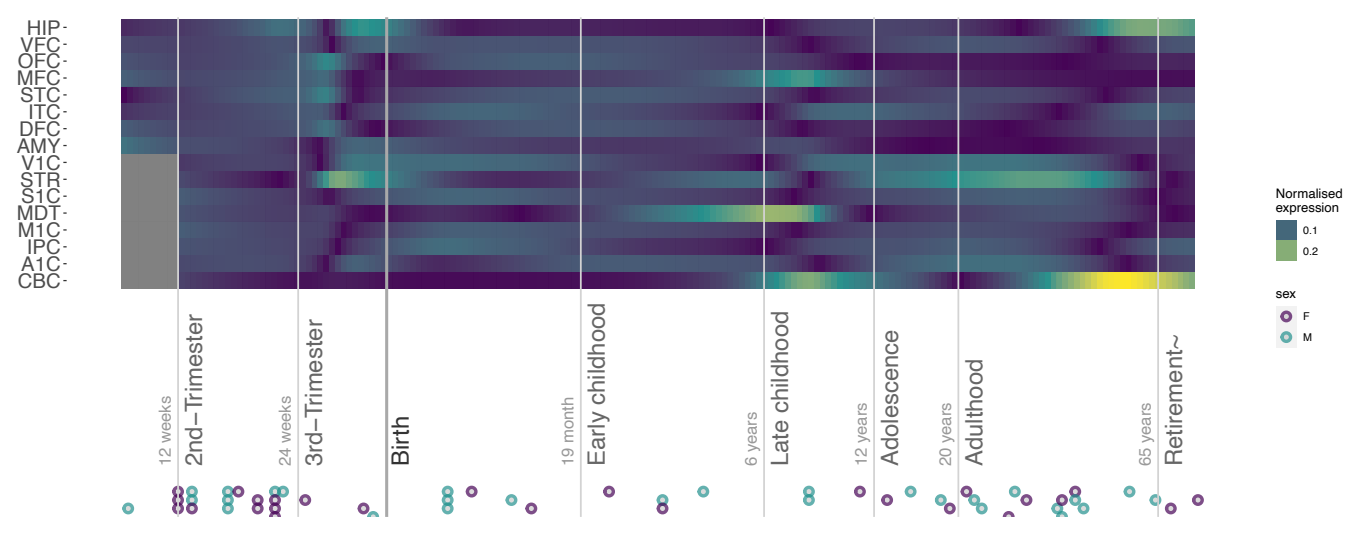

(b)

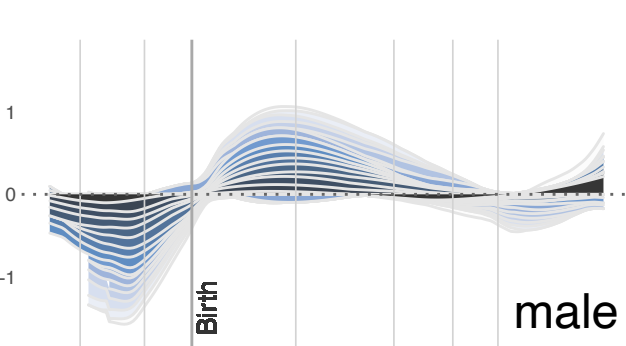

(c)

male

(d)

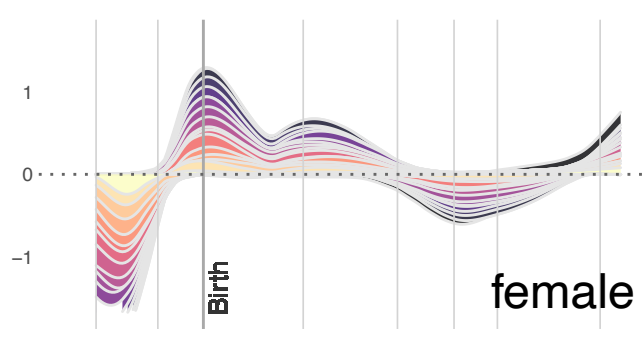

Figure 1. OXTR gene expression in sixteen regions of the human brain across different developmental phases. (a) The ribbon plot illustrates normalized gene expression compared to the lifespan average, with expression of individual brain regions stacked. The stacked Z-value is scaled to the number of regions (sixteen). (b) The heat plot illustrates gene expression across sixteen brain regions, normalized for each brain region across the lifespan. There is increased OXTR expression in the MFC, S1C, and CBC during late childhood, and increased expression in the STR and V1C during adulthood (see methods for a key to brain regions). Each individual donor and their sex are shown at the bottom of the panel. Time is presented using a log10 scale in both panels. Also presented are ribbon plots with data from only males (c) and females (d), with each panel illustrating normalized gene expression compared to the lifespan average. Males demonstrated a stronger early childhood peak compared to females, who demonstrated the highest OXTR expression just after birth.

in the striatum. Males exhibited a stronger early childhood peak in OXTR expression and more pronounced differentiation within brain regions (Figs. 1c and 1d; see Supplementary Figure 1 for greater detail). Expression patterns of $C D 38$, which regulates oxytocin secretion, and the structural gene for oxytocin (OXT) are presented in Supplementary figures 2 and 3. 


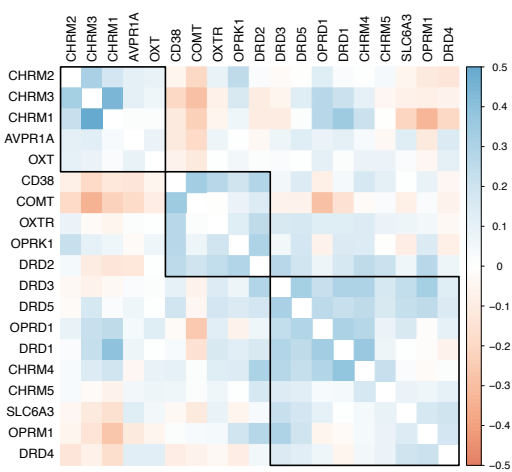

(a)

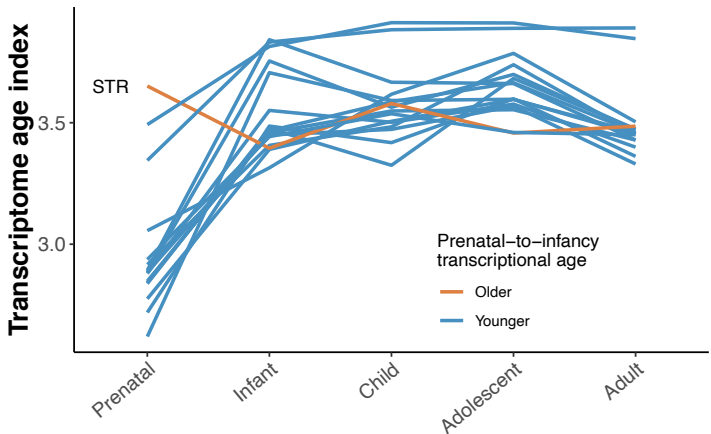

(d)

Life stage

(b)

c)
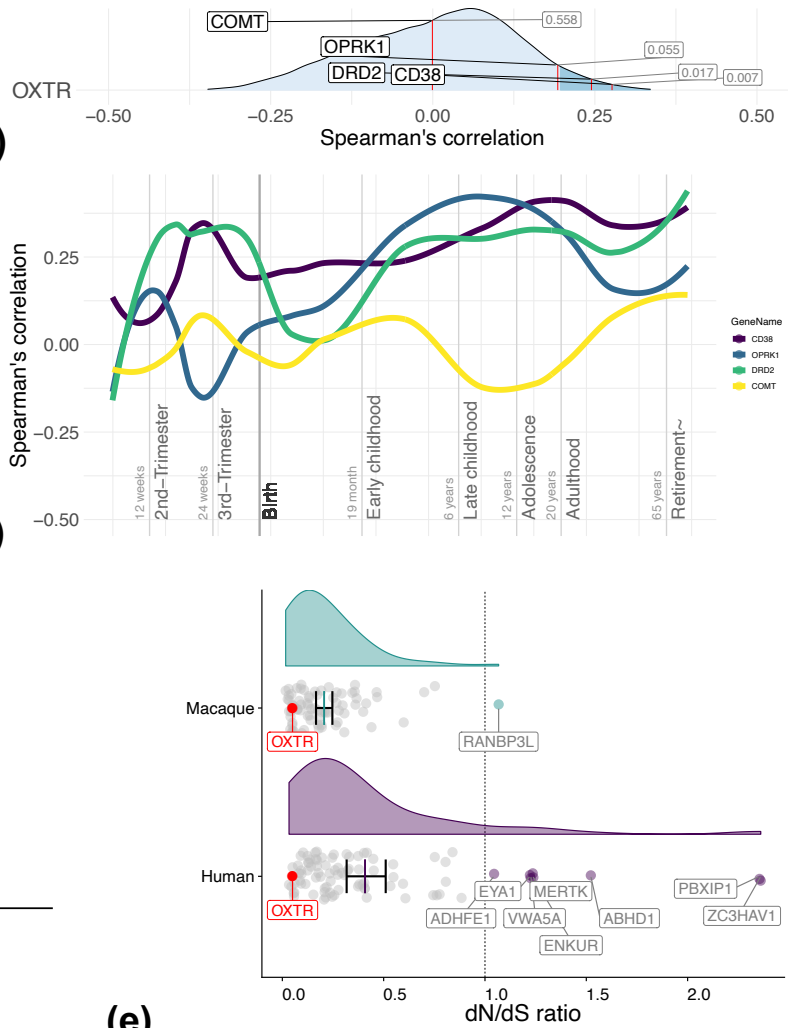

280

Figure 2. OXTR spatiotemporal co-expression and the evolution of OXTR co-expression modules. (a) The spatiotemporal correlation between OXTR and a selection of oxytocinergic, dopaminergic, muscarinic acetylcholine, and opioid pathway genes. OXTR was part of a five-gene spatiotemporal co-expression module with $D R D 2, C O M T$, OPRK1, and CD38. (b) The percentage rank of the spatiotemporal relationship between OXTR and DRD2, COMT, OPRK1, and CD38, compared with to the spatiotemporal relationship between OXTR and all protein coding genes ( $\mathrm{n}=16659$ ). Both $D R D 2$ and $C D 38$ were among with the top $5 \%$ of correlations (marked in dark blue). (c) The lifespan stability of the spatiotemporal correlation between OXTR and DRD2, COMT, OPRK1, CD38. (d) The transcriptional age of a gene co-expression module comprising OXTR and the 100 genes with the strongest spatiotemporal co-expression with OXTR across the lifespan in sixteen brain regions (see methods), with a smaller transcriptional age index representing an older transcriptome. Analysis suggested an older transcriptional age in infancy compared to the prenatal stage across the brain, except for the striatum (STR). (e) The top 100 gene module was more highly conserved in the macaque genome compared to the human genome $(p=0.0001)$, however, in both species OXTR (marked in red) is highly conserved. Several genes from this geneset had undergone positive selection in humans (i.e., dN/dS ratio values $>1$ ).

\section{Lifespan correlation between genes}

Hierarchical clustering identified a set of genes with particularly strong correlations for the spatiotemporal expression pattern of OXTR, which included CD38, COMT, OPRK1, and $D R D 2$ (Fig. 2a). Figure $2 \mathrm{~b}$ shows the distribution of the spatiotemporal correlations between 
(a)

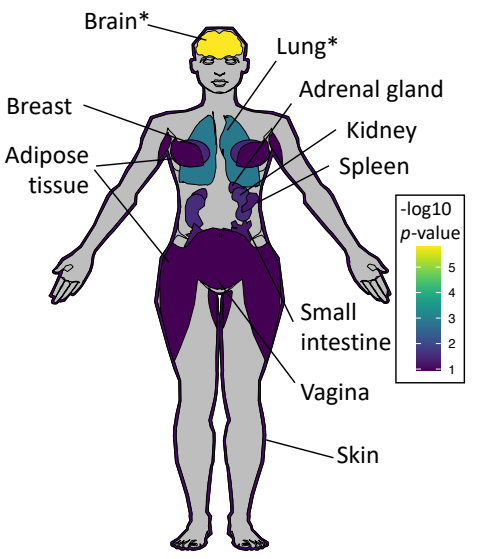

(b)

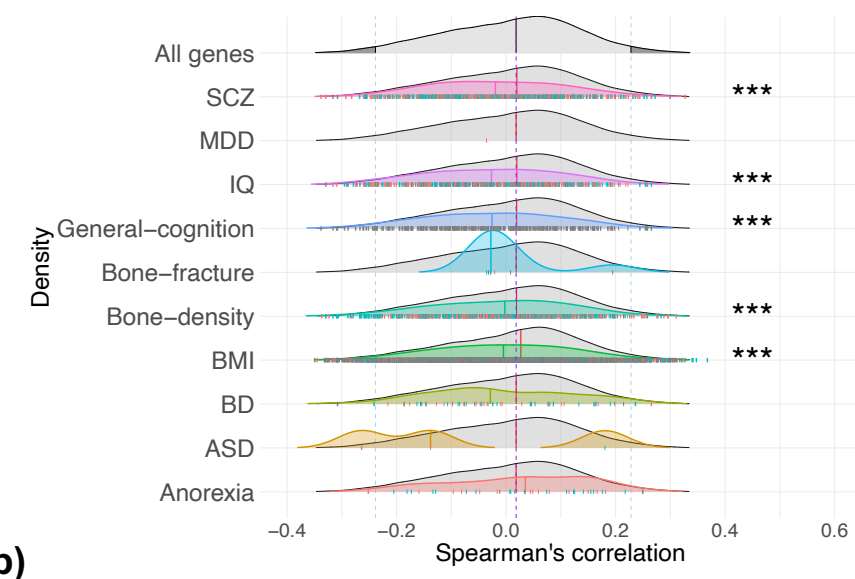

Figure 3. Networks of genes with strong spatiotemporal couplings with OXTR are enriched in brain and lung tissue, body composition phenotypes, and psychological phenotypes. (a) Top ten highest levels of differential expression in human tissue types of a geneset containing the top 100 genes correlated with OXTR. $\log 10 p$-values for upregulation of this geneset in various tissue types, represent the probability of the hypergeometric tests. See Supplementary figure 7 for a list of results with 30 tissue types. ${ }^{*} p<0.05$ (Bonferroni corrected). (b) Comparisons between the correlation of OXTR with genes related to disorders based on eQTL mapping and phenotypes and the correlation of OXTR with remaining genes. Vertical dashes in each distribution represent genes correlation between genes involved in phenotype and OXTR. Blue notches indicate risk increasing alleles which increase the expression of the gene, red notches indicate when risk increasing alleles decrease the expression of the gene, and grey notches indicate when direction is unknown, under respective distributions. ${ }^{* * *} p<0.001$ (FDR corrected).

OXTR and all remaining genes, marking the location of the genes clustered with OXTR. This analysis revealed that $D R D 2$ was among the top $1.7 \%$ of all correlated genes (total number of genes $=16660)$, and this correlation remained relatively stable from early childhood onward (Fig. 2c).

The evolutionary origin of OXTR and spatiotemporally related genes

As we observed in our human data analysis, a late-prenatal acceleration of OXTR expression was observed in macaques, however, there was no evidence of an early childhood peak (Supplementary Fig. 4). DRD2 was included in a cluster of genes with high spatiotemporal correlations with OXTR, but the relationship between OXTR and DRD2 was only in the top 
Table 1. Top 20 correlated and anti-correlated genes with OXTR across lifespan

\begin{tabular}{llllllll}
\hline & \multicolumn{3}{c}{ Top correlated } & & \multicolumn{3}{c}{ Top anti-correlated } \\
\cline { 2 - 3 } \cline { 6 - 7 } Rank & Gene & Trajectory & Arithmetic & & Gene & Trajectory & Arithmetic \\
\hline 1 & FGFR3 & 0.368 & 0.365 & & DEPDC5 & -0.381 & -0.365 \\
2 & GRAMD1C & 0.358 & 0.363 & & CACNA1A & -0.368 & -0.382 \\
3 & C1orf8 & 0.356 & 0.358 & & KLC2 & -0.363 & -0.373 \\
4 & NTSR2 & 0.35 & 0.318 & & KCNC3 & -0.363 & -0.313 \\
5 & INHBB & 0.348 & 0.328 & & C2CD3 & -0.361 & -0.319 \\
6 & CXorf59 & 0.343 & 0.348 & & HDAC8 & -0.359 & -0.345 \\
7 & KCNN3 & 0.341 & 0.336 & & CTCF & -0.349 & -0.3 \\
8 & DIRAS3 & 0.34 & 0.286 & & MAP3K12 & -0.347 & -0.356 \\
9 & STON2 & 0.338 & 0.345 & & MICAL2 & -0.345 & -0.313 \\
10 & RDH10 & 0.335 & 0.338 & & APBA2 & -0.345 & -0.285 \\
11 & AGXT2L1 & 0.328 & 0.301 & & MORC2 & -0.343 & -0.334 \\
12 & SIL1 & 0.327 & 0.293 & & NOVA1 & -0.341 & -0.318 \\
13 & HRSP12 & 0.326 & 0.316 & & NFIX & -0.34 & -0.321 \\
14 & GLUD1 & 0.323 & 0.309 & & KCNJ3 & -0.338 & -0.333 \\
15 & GNG12 & 0.323 & 0.32 & & KDM5C & -0.337 & -0.345 \\
16 & PBXIP1 & 0.321 & 0.304 & & SEMA4C & -0.333 & -0.315 \\
17 & RUNX3 & 0.319 & 0.289 & & EPS15L1 & -0.332 & -0.33 \\
18 & F3 & 0.317 & 0.312 & & TMEM25 & -0.33 & -0.325 \\
19 & ACSBG1 & 0.317 & 0.303 & & DHX8 & -0.33 & -0.307 \\
20 & GJA1 & 0.316 & 0.301 & & ADAM11 & -0.329 & -0.275 \\
\hline
\end{tabular}

Two approaches for this analysis are presented. "Trajectory" represents a mean correlation across the lifespan trajectory, in which each life period receives an equal weighting. "Arithmetic" represents the mean of individual correlations between donors, in which each donor is weighted equally.

A phylostratigraphic analysis revealed that most genes in a module containing genes with the 100 strongest spatiotemporal correlations with OXTR are evolutionary ancient,

327 appearing among the first three phylostrata (Supplementary Fig. 6). In particular, the ancestor of OXTR first emerged in the Eumetazoa phylostrata. A transcriptional age index (TAI) was calculated for sixteen brain regions across five ontogenetic stages, for which lower TAl values represent an older transcriptome. For all brain regions except the striatum (STR), the transcriptome of the OXTR top 100 geneset was older during the prenatal stage, compared to later stages (Fig. 2d). This suggests that genes in this transcriptome that are highly expressed

333 from infancy onward evolved at a faster rate compared to genes that are highly expressed prenatally, for most brain regions we investigated. 
Next, we calculated the $\mathrm{dN} / \mathrm{dS}$ ratio to assess whether genes in the top 100 geneset had experienced positive selection (dN/dS $>1)$, negative selection $(\mathrm{dN} / \mathrm{dS}<1)$, or if they have been evolving neutrally ( $\mathrm{dN} / \mathrm{dS} \sim 1$ ), in both the human and macaque genome (24). While OXTR is highly conserved in both humans and macaques, the total geneset on average had a significantly higher $\mathrm{dN} / \mathrm{dS}$ ratio in humans, compared to macaques $[t=3.96(123.3), p=$

$0.0001, d=0.6$; Fig. 2e]. This analysis also revealed that a number of genes show specific divergence in humans (i.e., positive selection), but not macaques, in which they are under selective constraint (ADHFE1, EYA1, MERTK, VWA5A, ENKUR, ABHD1, PBXIP1, and ZC3HAV1).

\section{The functional significance of spatiotemporally expression pattern}

Annotating the associations of a geneset including 100 genes with the strongest spatiotemporal associations with OXTR revealed enrichment in GWAS-derived genes for bone fracture in osteoporosis $\left(p=8.923 \times 10^{-3}\right)$ In addition, there was enrichment with genes associated with age-related macular degeneration GWAS $\left(p=8.293 \times 10^{-3}\right)$, as well as gene ontology genesets associated with reproduction $\left(p=5.83 \times 10^{-3}\right)$ and penile erection $(p=5.83$ $\left.x 10^{-3}\right)$. We also examined the enrichment of this geneset in thirty tissue types across the body using the GTEx database (version 8; (32)) in FUMA, discovering up-regulated differentially expressed genes in brain and lung tissue $(p>0.05$, Bonferroni corrected; Fig. 3a; Supplementary Fig. 7). The twenty genes showing the strongest spatiotemporal correlations with OXTR gene expression are presented in Table 1 . Some of the most positively correlated genes have been associated with bone mass regeneration (FGFR3) $(34,35)$, bone density (DIRAS3) (26), glucose (NTSR2) (36) and insulin allostasis (GLUD1) (37), and hypothalamic (INHBB) secretion (38). Some of the strongest negatively correlated genes have been associated with body mass index (MAP3K12, MORC2) (26) and bone density (CTCF, 
MORC2) (26). Notably, some of the highest correlated genes in this spatiotemporal analysis (e.g., NTSR2, GLUD1, SRPK1, MTCL1, KCNJ3, PAK7, THBS4, HEYL, ZC3HAV1) were also among the highest correlated in a previous analysis of OXTR expression in human adults using a different gene expression dataset (6).

To examine the relationship between the spatiotemporal expression of OXTR and genes that have been associated with psychological and physiological phenotypes of interest, we extracted phenotype genesets by using expression quantitative trait loci (eQTL) mapping of GWAS data (Supplementary table 1). To determine specificity, we compared these results with the relationship between spatiotemporal expression of OXTR and genes not included in the phenotype geneset of interest. The correlations with the genesets of interest and the background genes were both plotted as density distributions and non-parametric MannWhitney $U$ tests were performed to compare the distributions. There was a significantly lower density median for the relationship of OXTR with genesets enriched in schizophrenia, IQ, general cognition, bone density, and BMI, compared to the median of the density distribution with background genes, adjusting tests via a false discovery rate threshold $(p<0.001$; Fig. 3b; Supplementary Table 3). Genes related to phenotypes falling within top or bottom $2.5 \%$ of OXTR correlation's distribution are provided in Supplementary tables 4 and 5. Similar results were found using a MAGMA analysis (Supplementary Fig. 8; Supplementary Tables 6, 7, 8).

\section{Donor-to-donor reproducibility of gene expression patterns}

As the number of donor samples in these analyses is relatively small, it is important to confirm the stability of spatiotemporal gene expression patterns from donor-to-donor to make meaningful inferences beyond the sample. Thus, we calculated a measure of donor-to-donor spatiotemporal differential stability. Genes with a differential stability score in the top $50 \%$ of all genes are considered to be conserved (39). Previous work has demonstrated that genes with high differential stability have strong biological relevance (39) and that OXTR expression 


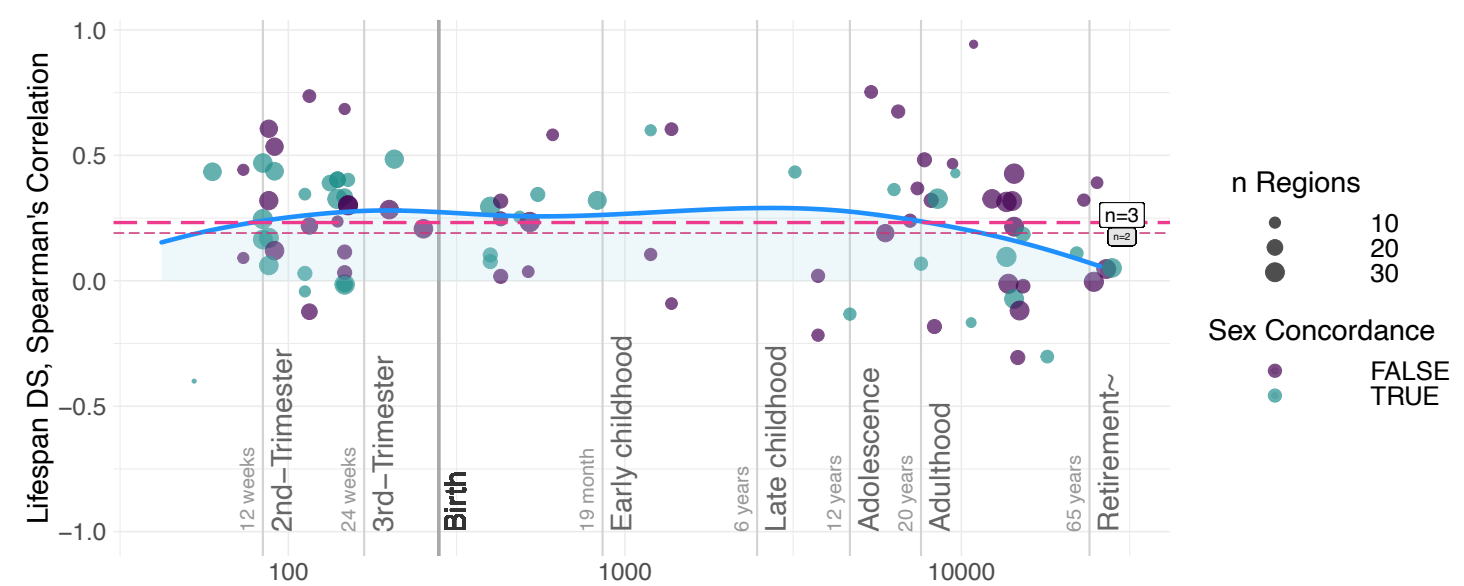

(a)
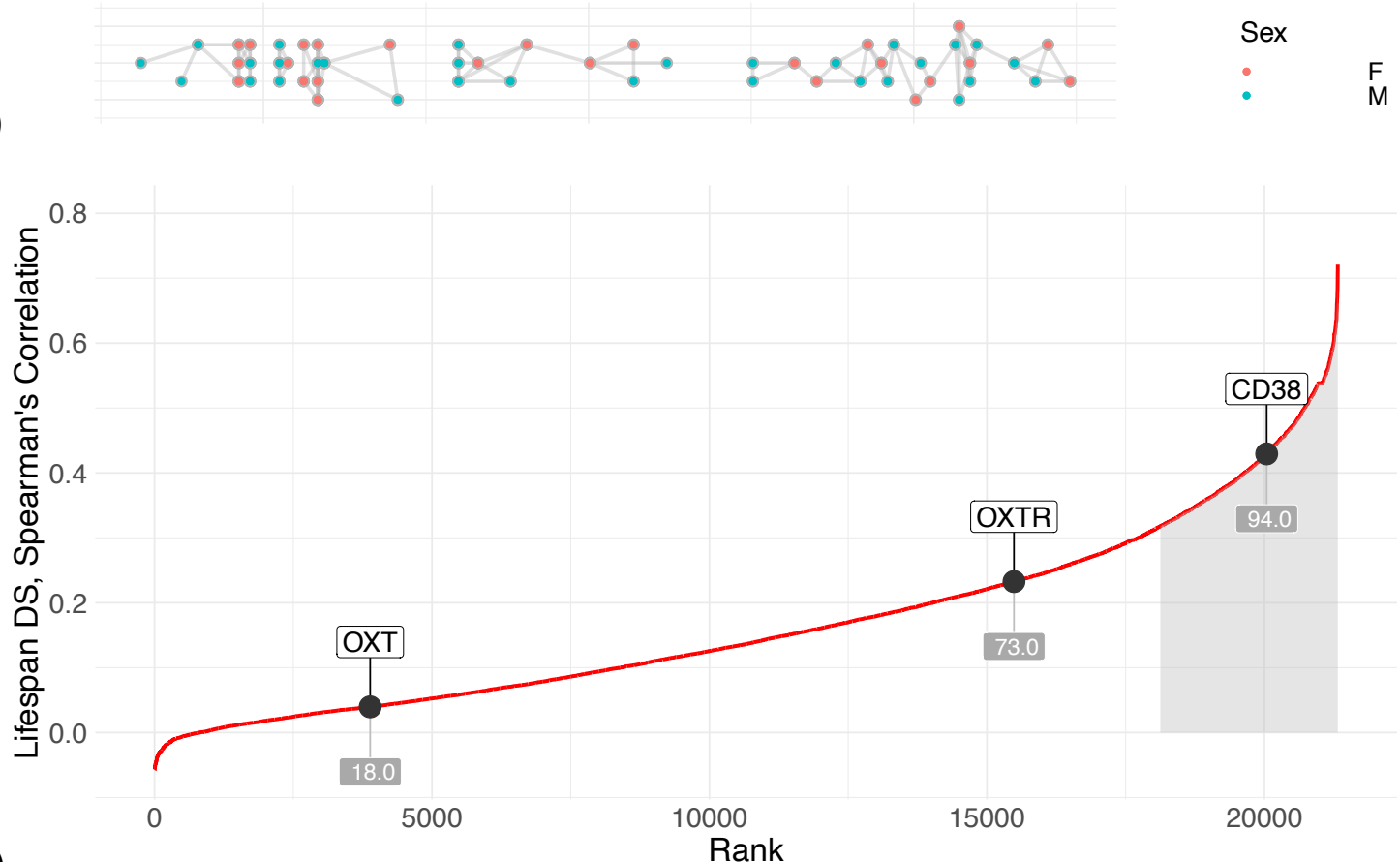

(b)

Figure 4. Differential stability for OXTR, CD38, and OXT across development. (a) The differential stability of OXTR is relatively constant across the lifespan, with a modest dip during adulthood. The blue line represents lifespan trajectory and the dashed horizontal red lines illustrate the mean of all points (long dashes $=3$ pair comparisons; short dashes $=4$ pair comparisons). Purple dots show the correlation between two donors of the same sex, whereas turquoise dots show the correlations of opposite sexes. The size of the dots corresponds to the number of brain regions. The bottom section illustrates a map of donor comparisons, where pairs used for differential stability estimation are connected with edges. (b) The differential stability of OXTR, CD38, and OXT were compared against 21,323 genes, across development. Genes in the top $15 \%$ of differential stability values are shaded in light grey. Both CD38 and OXTR can be considered to be highly conserved as their scores are in the top $50 \%$ (39). and $C D 38$ were among the top $30 \%$ of all protein coding genes in terms of spatiotemporal 
401 differential stability (Fig. 4). This indicates that expression patterns of these genes are 402 relatively stable in donors of a similar age. Differential stability of OXTR expression across development also remains relatively constant across macaque development (Supplementary Fig. 9).

\section{Discussion}

407 Here we characterized spatiotemporal gene expression patterns in human brain tissue, revealing increased OXTR expression during early childhood and a strong association between the spatiotemporal expression of OXTR and a key gene regulating dopaminergic signaling (DRD2). These features are conserved across individuals and appear to have emerged recently in human evolutionary history, as these gene expression patterns were not observed in a macaque sample. Moreover, we found that a geneset including genes with the strongest 100 associations with the OXTR spatiotemporal expression pattern is enriched in schizophrenia, IQ, general cognition, osteoporosis, reproductive processes, and BMI.

Our analyses revealed a distinct pattern of OXTR expression in the human brain across the lifespan, with an OXTR expression peak during childhood. This childhood peak was especially pronounced in males, which may contribute to reported sex differences in neurodevelopmental disorder diagnoses $(40,41)$. While the production and distribution of oxytocin in the brain is relatively similar across mammalian species (42), the location of oxytocin receptors in the brain varies between mammalian species both spatially $(43,44)$ and temporally (18). Indeed, differences in temporal expression peaks may reveal differing critical periods for experience-dependent development mediated by oxytocin signaling (45). For example, peak OXTR expression in mice occurs during the postnatal period $(46,47)$, which has also been shown to be a critical period for oxytocin-mediated cortical plasticity (48). Altogether, our observation of increased expression during early childhood highlights oxytocin's role during this critical life period in humans (11). 
We have previously demonstrated the strong co-expression of OXTR and dopaminergic signaling genes and the link between the OXTR expression pattern and brain regions associated with learning in the adult human brain (6). The strong spatiotemporal correlation between OXTR and DRD2 gene expression observed in the present study suggests that the oxytocin system works synergistically throughout development with the dopaminergic signaling to support learning (49), especially during critical developmental periods (2). We also demonstrated that OXTR is evolutionarily ancient, with its ancestor emerging around the Bilateria phylostrata during which basic nervous systems first appeared (50). However, the oxytocin system's integration with other signaling systems, and ultimately its purpose, seems to have shifted over time in response to novel environmental pressures (42). The increased integration of the oxytocin signaling system with the dopaminergic system in humans, compared to macaques, supports the critical importance of social learning in humans during early childhood (51).

In terms of the functional relevance of the OXTR spatiotemporal expression pattern, we found that this was highly correlated with a set of genes enriched for bone fracture. Moreover, several individual genes that were strongly associated with OXTR expression patterns have also been linked to bone integrity (i.e., FGFR3, DIRAS3, CTCF and MORC2). This is certainly not the first time the link between oxytocin signaling and bone remodeling has been highlighted (e.g., 52), however, the present results suggest that gene-gene coexpression in the brain may contribute to this association. Intriguingly, bone remodeling issues have been identified in autism (53), which has been associated with oxytocin signaling dysfunction (41), and oxytocin receptor knockout mice have been shown to develop osteoporosis (54). Moreover, peripheral oxytocin levels have been linked to bone mineral density in men with hypopituitarism (55) and post-menopausal women (56). Leptin release has been proposed as the primary central mediator of bone remodeling (57), but our results suggest that oxytocin may also play a critical role in this process. Although speculative, this 
453 finding points to a possible pleiotropic effect of oxytocin dysfunction on social difficulties and 454 bone remodeling, which warrants further investigation.

There are two limitations to the study worth noting. First, the donor sample sizes for both humans and macaques were relatively small. However, high differential stability values demonstrate that OXTR expression patterns were relatively stable from donor-to-donor in both human and macaque samples across the lifespan. Moreover, genes with high spatiotemporal co-expression with OXTR in the present study were also found to be highly co-expressed in our previous study in human adults, which used a different dataset for analysis (6). Second, we used transcriptome data as a proxy for gene expression density (19). While other methods directly measure OXTR expression density (e.g., competitive-binding receptor autoradiography), it is not practical to assess co-expression for more than a few receptors at a time using such approaches. While transcriptome measures are a less direct method, this facilitates the analysis of gene-gene co-expression patterns for thousands of receptor and non-receptor genes, which can help unravel the functional organization of the brain (39), for which oxytocin signaling was the focus in the present paper.

Here we provide evidence for distinct OXTR expression patterns that are enriched in psychological and body composition processes across development. These findings are consistent with the allostatic theory of oxytocin, which uniquely accounts for oxytocin's effects on both behavioral and non-behavioral traits and highlights the importance of oxytocin signaling function changes across the lifespan to adapt to shifting environmental challenges (2). By mapping the spatiotemporal OXTR gene expression pattern, identifying co-expressed genes, and better characterizing the evolutionary history of this pattern we provide evidence that oxytocin signaling is implicated in a broad suite of psychological and physiological

476 functions across the lifespan, and that this supporting role of the oxytocin system might be 477 unique to humans. 


\section{Acknowledgements}

480 This research was funded by the Research Council of Norway (301767), the Novo Nordisk

481 Foundation (NNF16OC0019856), and the ERA-Net Cofund through the ERA PerMed project 482 "IMPLEMENT".

483 
485 The authors have no competing interests to disclose.

486 


\section{References}

488

1. Jurek B, Neumann ID (2018): The oxytocin receptor: from intracellular signaling to behavior. Physiol Rev 98: 1805-1908.

2. Quintana DS, Guastella AJ (2020): An allostatic theory of oxytocin. Trends Cogn Sci 24: 515-528.

3. Busnelli M, Chini B (2018): Molecular Basis of Oxytocin Receptor Signalling in the Brain: What We Know and What We Need to Know. Curr Top Behav Neurosci 35: 3-29.

4. Keebaugh AC, Barrett CE, Laprairie JL, Jenkins JJ, Young $\amalg$ (2015): RNAi knockdown of oxytocin receptor in the nucleus accumbens inhibits social attachment and parental care in

5. Nishimori K, Takayanagi Y, Yoshida M, Kasahara Y, Young L, Kawamata M (2008): New aspects of oxytocin receptor function revealed by knockout mice: sociosexual behaviour and control of energy balance ((I. D. Neumann \& R. Landgraf, editors)). Prog Brain Res 170: 79-90.

6. Quintana DS, Rokicki J, van der Meer D, Alnæs D, Kaufmann T, Córdova-Palomera A, et al. (2019): Oxytocin pathway gene networks in the human brain. Nat Commun 10: 668.

7. Lee MR, Sheskier MB, Farokhnia M, Feng N, Marenco S, Lipska BK, Leggio L (2018): Oxytocin receptor mRNA expression in dorsolateral prefrontal cortex in major psychiatric disorders: A human post-mortem study. Psychoneuroendocrinology 96: 143-147.

8. Uhrig S, Hirth N, Broccoli L, von Wilmsdorff M, Bauer M, Sommer C, et al. (2016): Reduced oxytocin receptor gene expression and binding sites in different brain regions in

9. Kang HJ, Kawasawa YI, Cheng F, Zhu Y, Xu X, Li M, et al. (2011): Spatiotemporal transcriptome of the human brain. Nature 478: 483-489.

10. Kim DR, Bale TL, Epperson CN (2015): Prenatal programming of mental illness: current understanding of relationship and mechanisms. Curr Psychiatry Rep 17: 5. 
11. Freeman SM, Palumbo MC, Lawrence RH, Smith AL, Goodman MM, Bales KL (2018): Effect of age and autism spectrum disorder on oxytocin receptor density in the human basal forebrain and midbrain. Transl Psychiatry 8: 1-11.

12. Boer GJ, Swaab DF, Uylings HBM, Boer K, Buijs RM, Velis DN (1980): Neuropeptides in Rat Brain Development. In: McConnell PS, Boer GJ, Romijn HJ, Van De Poll NE, Corner MA, editors. Progress in Brain Research, vol. 53. Elsevier, pp 207-227.

13. Swaab DF (1995): Development of the human hypothalamus. Neurochem Res 20: 509-519.

14. Pedersen CA, Boccia ML (2002): Oxytocin links mothering received, mothering bestowed and adult stress responses. Stress Amst Neth 5: 259-267.

15. Winslow JT, Noble PL, Lyons CK, Sterk SM, Insel TR (2003): Rearing effects on cerebrospinal fluid oxytocin concentration and social buffering in rhesus monkeys. Neuropsychopharmacology 28: 910-918.

16. Francis DD, Champagne FC, Meaney MJ (2000): Variations in maternal behaviour are associated with differences in oxytocin receptor levels in the rat. J Neuroendocrinol 12: 1145-1148.

17. Heim C, Young L, Newport DJ, Mletzko T, Miller AH, Nemeroff CB (2009): Lower CSF oxytocin concentrations in women with a history of childhood abuse. Mol Psychiatry 14: 954-958.

18. Vaidyanathan R, Hammock EA (2017): Oxytocin receptor dynamics in the brain across development and species. Dev Neurobiol 77: 143-157.

19. Young L, Muns S, Wang Z, Insel TR (1997): Changes in oxytocin receptor mRNA in rat brain during pregnancy and the effects of estrogen and interleukin-6. J Neuroendocrinol 9: 859865.

20. Domazet-Lošo T, Tautz D (2010): A phylogenetically based transcriptome age index mirrors ontogenetic divergence patterns. Nature 468: 815-818.

21. Hawrylycz M, Lein ES, Guillozet-Bongaarts AL, Shen EH, Ng L, Miller JA, et al. (2012): An anatomically comprehensive atlas of the adult human brain transcriptome. Nature 489: 391. 
22. Domazet-Lošo T, Tautz D (2008): An Ancient Evolutionary Origin of Genes Associated with Human Genetic Diseases. Mol Biol Evol 25: 2699-2707.

23. Drost H-G, Gabel A, Liu J, Quint M, Grosse I (2018): myTAl: evolutionary transcriptomics with R. Bioinformatics 34: 1589-1590.

24. Dumas G, Malesys S, Bourgeron T (2021): Systematic detection of brain protein-coding genes under positive selection during primate evolution and their roles in cognition. Genome Res 31: 484-496.

25. Watanabe K, Taskesen E, Bochoven A, Posthuma D (2017): Functional mapping and annotation of genetic associations with FUMA. Nat Commun 8: 1826.

26. MacArthur J, Bowler E, Cerezo M, Gil L, Hall P, Hastings E, et al. (2017): The new NHGRI-EBI Catalog of published genome-wide association studies (GWAS Catalog). Nucleic Acids Res 45: D896-D901.

27. Liberzon A, Subramanian A, Pinchback R, Thorvaldsdóttir H, Tamayo P, Mesirov JP (2011): Molecular signatures database (MSigDB) 3.0. Bioinformatics 27: 1739-1740.

28. Kerimov N, Hayhurst JD, Peikova K, Manning JR, Walter P, Kolberg L, et al. (2021): eQTL Catalogue: a compendium of uniformly processed human gene expression and splicing QTLs. bioRxiv 2020.01.29.924266.

29. Akbarian S, Liu C, Knowles JA, Vaccarino FM, Farnham PJ, Crawford GE, et al. (2015): The PsychENCODE project. Nat Neurosci 18: 1707-1712.

30. Ng B, White CC, Klein H-U, Sieberts SK, McCabe C, Patrick E, et al. (2017): An xQTL map integrates the genetic architecture of the human brain's transcriptome and epigenome. Nat Neurosci 20: $1418-1426$.

31. Hoffman GE, Bendl J, Voloudakis G, Montgomery KS, Sloofman L, Wang Y-C, et al. (2019): CommonMind Consortium provides transcriptomic and epigenomic data for Schizophrenia and Bipolar Disorder. Sci Data 6: 180. 
32. Aguet F, Brown AA, Castel SE, Davis JR, He Y, Jo B, et al. (2017): Genetic effects on gene expression across human tissues. Nature 550: 204-213.

33. Ramasamy A, Trabzuni D, Guelfi S, Varghese V, Smith C, Walker R, et al. (2014): Genetic variability in the regulation of gene expression in ten regions of the human brain. Nat Neurosci 17: 1418-1428.

34. Valverde-Franco G, Liu H, Davidson D, Chai S, Valderrama-Carvajal H, Goltzman D, et al. (2004): Defective bone mineralization and osteopenia in young adult FGFR3-/- mice. Hum Mol Genet 13: 271-284.

35. Mugniery E, Dacquin R, Marty C, Benoist-Lasselin C, de Vernejoul M-C, Jurdic P, et al. (2012): An activating Fgfr3 mutation affects trabecular bone formation via a paracrine mechanism during growth. Hum Mol Genet 21: 2503-2513.

36. Mazella J, Béraud-Dufour S, Devader C, Massa F, Coppola T (2012): Neurotensin and its receptors in the control of glucose homeostasis. Front Endocrinol 3. https://doi.org/10/gft367

37. Tanizawa Y, Nakai K, Sasaki T, Anno T, Ohta Y, Inoue H, et al. (2002): Unregulated Elevation of Glutamate Dehydrogenase Activity Induces Glutamine-Stimulated Insulin Secretion: Identification and Characterization of a GLUD1 Gene Mutation and Insulin Secretion Studies With MIN6 Cells Overexpressing the Mutant Glutamate Dehydrogenase. Diabetes 51: 712717.

38. Ying S-Y (1987): Inhibins and Activins: Chemical Properties and Biological Activity. Proc Soc Exp Biol Med 186: 253-264.

39. Hawrylycz M, Miller JA, Menon V, Feng D, Dolbeare T, Guillozet-Bongaarts AL, et al. (2015): Canonical genetic signatures of the adult human brain. Nat Neurosci 18: 1832.

40. Ferri SL, Abel T, Brodkin ES (2018): Sex Differences in Autism Spectrum Disorder: a Review. Curr Psychiatry Rep 20: 9.

41. Guastella AJ, Hickie IB (2016): Oxytocin treatment, circuitry and autism: a critical review of the literature placing oxytocin into the autism context. Biol Psychiatry 79: 234-242. 
42. Knobloch HS, Grinevich V (2014): Evolution of oxytocin pathways in the brain of vertebrates. Front Behav Neurosci 8: 31.

43. Leung CH, Abebe DF, Earp SE, Goode CT, Grozhik AV, Mididoddi P, Maney DL (2011): Neural distribution of vasotocin receptor mRNA in two species of songbird. Endocrinology 152: 4865-4881.

44. Freeman SM, Young L (2016): Comparative Perspectives on Oxytocin and Vasopressin Receptor Research in Rodents and Primates: Translational Implications. J Neuroendocrinol 28. https://doi.org/10.1111/jne.12382

45. Hammock E (2015): Developmental perspectives on oxytocin and vasopressin. Neuropsychopharmacology 40: 24.

46. Mitre M, Marlin BJ, Schiavo JK, Morina E, Norden SE, Hackett TA, et al. (2016): A Distributed Network for Social Cognition Enriched for Oxytocin Receptors. J Neurosci 36: 2517-2535.

47. Hammock E, Levitt P (2013): Oxytocin receptor ligand binding in embryonic tissue and postnatal brain development of the C57BL/6J mouse. Front Behav Neurosci 7: 195.

48. Zheng J-J, Li S-J, Zhang X-D, Miao W-Y, Zhang D, Yao H, Yu X (2014): Oxytocin mediates early experience-dependent cross-modal plasticity in the sensory cortices [no. 3]. Nat Neurosci 17: 391-399.

49. Love TM (2014): Oxytocin, motivation and the role of dopamine. Pharmacol Biochem Behav 119: $49-60$.

50. Kelava I, Rentzsch F, Technau U (2015): Evolution of eumetazoan nervous systems: insights from cnidarians. Philos Trans R Soc B Biol Sci 370: 20150065.

51. Nielsen M (2012): Imitation, pretend play, and childhood: Essential elements in the evolution of human culture? J Comp Psychol 126: 170-181.

52. Copland JA, Ives KL, Simmons DJ, Soloff MS (1999): Functional Oxytocin Receptors Discovered In Human Osteoblasts. Endocrinology 140: 4371-4374. 
53. Hediger ML, England L, Molloy CA, Yu KF, Manning-Courtney P, Mills JL (2008): Reduced Bone Cortical Thickness in Boys with Autism or Autism Spectrum Disorder. J Autism Dev Disord 38: $848-856$.

54. Tamma R, Colaianni G, Zhu L, DiBenedetto A, Greco G, Montemurro G, et al. (2009): Oxytocin is an anabolic bone hormone. Proc Natl Acad Sci 106: 7149-7154.

55. Aulinas A, Guarda FJ, Yu EW, Haines MS, Asanza E, Silva L, et al. (2021): Lower Oxytocin Levels Are Associated with Lower Bone Mineral Density and Less Favorable Hip Geometry in Hypopituitary Men. Neuroendocrinology 111: 87-98.

56. Breuil V, Panaia-Ferrari P, Fontas E, Roux C, Kolta S, Eastell R, et al. (2014): Oxytocin, a New Determinant of Bone Mineral Density in Post-Menopausal Women: Analysis of the OPUS Cohort. J Clin Endocrinol Metab 99: E634-E641.

57. Ducy P, Amling M, Takeda S, Priemel M, Schilling AF, Beil FT, et al. (2000): Leptin Inhibits Bone Formation through a Hypothalamic Relay: A Central Control of Bone Mass. Cell 100: 197207. 